\title{
Féminisme de marché et égalité élitiste ?
}

\section{Sophie Pochic}

Un des faits marquants des décennies 2000 et 2010 est que l'égalité entre les femmes et les hommes est devenue un objet d'investissement des grandes entreprises, qui en font un enjeu d'image et d'attractivité. C'est évidemment lié à l'impulsion des politiques publiques, avec des lois plus contraignantes sur l'égalité professionnelle et la lutte contre les discriminations depuis 2001 en France. Cette avancée repose aussi sur la mobilisation des femmes elles-mêmes, notamment des plus diplômées, qui ont réussi à faire des inégalités au sommet et du "plafond de verre " un sujet de débat public dans les années 2000, dans le sillage de la loi sur la parité en politique. Les idées féministes semblent regagner du terrain dans la presse féminine, et même pénétrer la presse économique et le monde des affaires. Je propose d'appeler féminisme de marché cette nébuleuse d'actrices et d'initiatives privées, qui se réalise à distance des associations féministes et des syndicats, traditionnellement situés à gauche. Si ce courant a indéniablement relancé les débats et les actions sur la mixité au travail, il comporte le risque d'une " égalité élitiste " réservée à une minorité de femmes de talent et donc d'accentuer la polarisation au sein des femmes.

\section{Le féminisme de marché, un nouveau courant transnational ?}

Dans les années 2000, une mobilisation collective s'est organisée contre le " plafond de verre " et pour la parité dans le monde économique en France. Elle a fait advenir la loi Zimmermann-Copé de 2011, imposant à terme des quotas de $40 \%$ de femmes dans les conseils d'administration et les conseils de surveillance des grandes entreprises. Cette mobilisation pour une "représentation équilibrée " dans les espaces de décision a mis au jour de nouveaux groupes d'intérêts, les réseaux féminins (de cadres), identifiés comme de "nouveaux acteurs de l'égalité professionnelle" [Laufer, 2014]. Ces réseaux se sont structurés autour de grandes entreprises et de grandes écoles parisiennes, à l'initiative de consultantes et de femmes d'affaires [Boni-Legoff, 2010 ; Blanchard, Boni-Legoff et Rabier, 2013]. Les succès scolaires des filles, particulièrement dans les écoles de commerce, et leur entrée massive dans les métiers qualifiés, sont une dimension explicative sous-jacente. Elles ont ainsi atteint, puis dépassé, le seuil numérique de $30 \%$ leur permettant de questionner les privilèges du groupe majoritaire, si l'on reprend l'analyse de Rosabeth Moss Kanter [Kanter, 1977]. Leur accès privilégié aux médias et au web, en tant qu'actrices, témoins ou expertes de l'égalité, a contribué fortement à populariser leur cause. Mais ce relais par les journalistes (elles-mêmes cadres) fait parfois surestimer leur poids dans la population active : les femmes "cadres et professions intellectuelles supérieures " sont en croissance, 
certes, mais elles ne représentent encore que $14,7 \%$ des femmes en emploi en France en 2015.

En déclinant le concept de "l'espace de la cause des femmes " [Bereni, 2015], Marion Rabier a dénommé cette nébuleuse réformatrice mobilisée pour la parité économique "l'espace de la cause des dirigeantes ». Ce concept souligne l'aspect transsectoriel de cette cause, porté par des collectifs organisés au nom des femmes et pour les femmes, qui circule au sein de pôles sociaux souvent séparés dans les enquêtes de sciences sociales: entreprises, associations, administrations, partis politiques et intellectuels [Rabier, 2013]. Mais la métaphore spatiale laisse à penser que cet espace est traversé d'argumentaires idéologiques variés. Plus encore, elle semble sous-entendre que cette cause serait limitée à nos frontières nationales, alors que ce discours se retrouve dans des aires culturelles variées et au niveau international.

Nous préférons appeler cette nébuleuse le "féminisme de marché », en dialogue avec des auteures anglo-saxonnes, afin d'en souligner à la fois le substrat idéologique commun et la dimension transnationale. Les appellations de ce courant diffèrent selon les auteures: "bâtard néolibéral du féminisme " [Fraser, 2011], " managerial feminism " [Eisenstein, 2009], " market feminism » [Kantola et Squires, 2012], " transnational business feminism » [Robert, 2012] ou "néolibéralisation du féminisme " [Prügl, 2014]. Ces notions désignent des discours pragmatiques et technicisés sur les bénéfices économiques de l'égalité entre les femmes et les hommes que portent les directions de multinationales et les majors du conseil, repris par des organisations internationales (Nations unies, Fonds monétaire international, Banque mondiale, Union européenne) et relayés par des organisations non gouvernementales (ONG) dans des pays en voie de développement. Quelle que soit leur définition, ces auteures s'accordent sur le fait que ces discours ont en commun de valoriser le marché, l'entrepreneuriat et les capacités individuelles (l'empowerment) comme moyens d'améliorer la condition des femmes, et de négliger les causes structurelles et historiques des inégalités et de la division sexuée du travail.

Nommer "féminisme de marché " cette rhétorique permet de souligner d'une part qu'elle ne comporte aucune dimension critique sur les effets sociaux du capitalisme financier. Les majors du conseil en stratégie et audit (Ernst \& Young, McKinsey, Accenture, Deloitte) sont les véhicules de cette idée de cercle vertueux : I'égalité serait bonne pour le marché, et le marché serait bon pour l'égalité. Ces discours naviguent entre sphère privée et sphère publique, des cabinets de conseil aux administrations, et promeuvent cette " égalité pour le marché " [Jacquot, 2014] ou cette " égalité sous condition de performance " [Sénac, 2015]. Cette doxa se présente comme apolitique, mais légitime, voire encense l'action des grandes entreprises, qui seraient l'avant-garde éclairée de l'égalité entre les femmes et les hommes. Elle ne se conçoit pas sur le modèle de la démocratie participative, où des femmes représentant les intérêts et les " voix " de toutes, notamment des moins dotées, pourraient discuter l'orientation des politiques et les résultats des actions. Cela explique sans doute 
pourquoi cette approche qui fait des patrons les principaux " champions du changement " est développée prioritairement dans des pays très libéraux, comme l'Australie et les ÉtatsUnis, notamment par un think-tank féministe-libéral financé par des multinationales américaines (Catalyst) et relayée en France par le comité « diversité » du Medef.

Ce courant prône aussi des techniques néolibérales de gouvernement [Prügl, 2014]. Des dispositifs de gestion de la diversité relativement standardisés, inventés pour des multinationales américaines, sont désormais préconisés dans le monde entier pour toutes les organisations, publiques ou privées : monitoring (suivi d'indicateurs chiffrés), networking (réseaux de femmes cadres), mentoring et coaching et enfin dispositifs de work-life balance (horaires aménagés, participation financière de l'entreprise aux frais de garde ou aux congés pour raisons familiales). Cette rhétorique est compatible, et même promeut, des dispositifs individualisés et sélectifs pour une minorité de salariées ou d'entrepreneuses "à potentiel », " de talent " ou "d'excellence ", traitées à part de la masse des travailleuses ordinaires. En tant qu'employeurs, les majors du secteur du conseil et de l'audit, confrontés à une forte déperdition de femmes cadres en raison d'un temps de travail extensif et d'une intense compétition interne se l'appliquent souvent à eux-mêmes [Boni-Legoff, 2013]. Ces cabinetsconseils sont aussi les chantres du libéralisme économique et des bénéfices de la mondialisation, et emploient l'élite sociale issue des business schools de chaque pays où ils sont implantés [Djelic, 2004]. Plus largement, les multinationales cotées en bourse doivent aujourd'hui répondre aux attentes du "marché " sur leur Responsabilité sociale et environnementale (RSE), en publiant des indicateurs quantifiables de performance sociale et environnementale, dont des indicateurs sexués [Bender, Berrebi-Hoffmann et Reigné, 2015].

Cette forme de néolibéralisation du féminisme [Prügl, 2014], peut aussi être caractérisée par ce qu'elle n'autorise pas, ou n'intègre pas, ce qui révèle en creux sa spécificité. D'une part, elle assume la sélectivité sociale des participant·e·s, avec des évènements, dispositifs et formations réservées dans des lieux de la bourgeoisie des affaires. On n'y trouve quasiment jamais un ou une syndicaliste, ni dans la salle, et surtout pas à la tribune. Si le Women's Forum de Deauville, ou le programme Women Leaders and Gender Parity du Forum de Davos, en sont deux illustrations emblématiques, la plupart des évènements organisés par les réseaux féminins et/ou des consultant·e.s, fonctionnent sur le même principe de clôture sociale des participant.e.s et des discours. Ces lieux sont autant le moyen de conscientiser des femmes cadres supérieures et de partager dans un entre-soi feutré les « bonnes pratiques » entre organisations, qu'une vitrine pour mettre en valeur les grandes entreprises, leurs politiques de ressources humaines et leurs produits. Cette rhétorique ne comporte quasiment aucune remise en question des règles du jeu du capitalisme financier, à part qu'il devrait désormais se parer de rose...

Mais il faut aussi reconnaître l'efficacité pratique de ce courant porté par des femmes d'influence, dont certaines spécialisées en marketing, lobbying ou communication, et avec les moyens des puissantes multinationales, leurs employeurs ou partenaires. Parler des 
bénéfices économiques de l'égalité, plutôt que de valeurs morales, de contraintes légales ou de risques juridiques, est une stratégie gagnante qui a réussi à convaincre des dirigeants réticents face aux contraintes ou aux coûts que représentent de telles actions positives [Bereni, 2009]. Le contexte de crise financière et économique majeure depuis 2008 a paradoxalement favorisé la réception sur les bénéfices du « management au féminin » face aux dérives masculinistes viriles du monde de la finance [Blanchard, Boni et Rabier, 2013]. En outre, le registre différentialiste des "qualités féminines" permet à des patrons de se présenter comme des "hommes modernes", sans interroger leur position de dominants dans les rapports de pouvoir entre hommes et femmes et en faisant l'économie d'un questionnement sur leurs propres pratiques professionnelles ou privées [Connell, 2005]. Le registre "pur» de la performance (renforcer la mixité des équipes commerciales, de Recherche et Développement ou du design, en raison de leurs meilleurs résultats) serait parfois le seul moyen de vaincre sans brusquer les résistances des cadres masculins aux actions positives " pour femmes ».

Au-delà de discours critiques parfois moralisateurs ou manichéens, parfois teintés de nostalgie, portés par des théoriciennes féministes, il me semble urgent d'étudier les effets concrets de cette rhétorique qui se développe à distance de l'égalité négociée et des organisations syndicales.

\section{Le cadrage élitiste de la " gestion de la diversité »}

Quels sont les effets de cette rhétorique du "féminisme de marché " au sein des grandes entreprises en France? Les réseaux de femmes (cadres) et les organisations syndicales peuvent être analysés comme des groupes d'intérêts aux ressources asymétriques, qui ne portent pas le même cadrage de l'égalité, qui n'ont pas les mêmes prérogatives et qui ne représentent pas les mêmes groupes. En Angleterre, Cynthia Cockburn souligne dès les années 1980 que les politiques d'égalité des chances (equal opportunity) peuvent avoir une ambition transformatrice de long terme, trait qu'elle observe plutôt dans le secteur public: changer la structure du pouvoir et de la hiérarchie au sein même des organisations, avec des groupes non-mixtes et des syndicats engagés sur les droits des femmes les moins dotées. Ces politiques se restreignent souvent à une version managériale légère de court terme, apanage du secteur privé : nettoyage des procédures formelles de leurs biais discriminatoires et attention focalisée sur les femmes managers [Cockburn, 1991]. Une récente ethnographie d'une grande entreprise aux États-Unis [Berrey, 2014] confirme qu'au-delà des principes et des méthodes, les programmes de gestion de la diversité des grandes entreprises sont sélectifs en termes d'ayants droit. Alors que la lutte contre les discriminations (equal opportunity) concerne en principe tou·te-s les salarié.e.s, la gestion de la diversité (diversity management) est réservée aux seuls " hauts potentiels » issus de deux catégories : les femmes et les minorités ethniques. Ce prisme managérial de la diversité bonne pour la performance a pour effet de consolider la hiérarchie et de négliger 
les salarié·e·s du bas de l'échelle, ceux qu'Ellen Berrey désigne comme le « sale plancher " (dirty floor).

Le féminisme de marché tend donc à concentrer l'action des directions sur le sommet de la hiérarchie, avec le risque de générer une " égalité élitiste " réservée en priorité à une minorité de femmes très dotées [Jacquemart et al., 2016]. C'est aussi souvent une vision de l'égalité statique et numérique, se préoccupant surtout de la coq présence (ou de l'absence) des femmes de bastions professionnels masculins, en négligeant la dynamique de production des inégalités de parcours et de condition, par les organisations ellesq mêmes, tout au long du cycle de vie professionnelle. Elle passe sous silence leur imbrication avec d'autres rapports sociaux de pouvoir (de classe, de race et de sexualité notamment), elle tend à re naturaliser la catégorie des femmes autour de l'expérience partagée de la maternité et questionne peu le "régime d'inégalités» des organisations [Acker, 2009]. Le féminisme de marché est donc très éloigné, socialement et idéologiquement, du féminisme ouvrierq syndical, qui justifie les droits des femmes au nom de la justice sociale [Maruani, 1979], ou du féminisme d'extrême gauche, que les Anglo Saxonnes désignent comme un radical feminism, irrigué par les mouvements féministes lesbiens et le black feminism. Les réseaux féminins, sélectifs socialement et au registre policé, sont relativement écoutés et soutenus par les directions des grandes entreprises.

Les syndicats se méfient souvent des initiatives portées par ces réseaux de femmes cadres, rarement syndiquées, et à distance de la négociation collective. En France, les syndicats sont souvent critiqués pour leur distance (réciproque) à l'égard du mouvement féministe et leur rapport relativement défensif aux questions d'égalité. Mais quelles marges de manœuvre les élu·e.s de comité d'entreprise ont- ils et ont- elles pour modifier des dispositifs proposés par la direction? Et de quelle formation, informations et expertise disposent- ils/elles pour ouvrir un débat démocratique sur des sujets complexes et parfois conflictuels comme l'égalité salariale? Les syndicats français sont des colosses aux pieds d'argile, qui fonctionnent avec très peu d'adhérents, peu de droits syndicaux dans les petites et moyennes entreprises (PME) et autour d'une petite poignée de militants. Ces derniers ont rarement été formés à endosser des " lunettes genre " pour intégrer les questions femmes - égalité - discriminations dans l'ensemble des négociations, et ont de plus souvent des marges de manœuvres assez faibles pour modifier le contenu même des accords [Cristofalo, 2013 ; Laufer et Silvera, 2017]. Les militantes engagées sur l'égalité ne sont pas toujours soutenues par les collectifs syndicaux mixtes [Guillaume, 2018], car leurs camarades syndicalistes sont souvent convaincus par le mythe de "l'égalitéq déjà- là » [Delphy, 2001]. En outre, elles peuvent être aussi déçues par le contenu même des accords négociés, relativement standardisés, avec peu d'actions concrètes et des budgets priorisés autour de la promotion des femmes cadres et de la mixité des métiers masculins, au détriment d'autres dossiers comme la précarité de l'emploi, la pénibilité du travail ou les horaires atypiques et bien sûr les inégalités salariales à tous les échelons [Charpenel et al., 2017]. Enfin les syndicats peuvent être traversés par des débats, entre des femmes cadres désormais conscientisées sur le sexisme ordinaire et des 
femmes non-cadres qui ne se sentent pas discriminées " en tant que femmes " dans des services majoritairement féminins.

\section{Jeux d'échelle autour de la dualisation du marché du travail féminin}

Quand une multinationale communique sur la mixité de sa gouvernance, utile pour son image de marque et sa cotation sociale, elle ne dit rien des conditions de travail et de vie au travail, notamment des femmes non- cadres [Kirton et Greene, 2005]. L'hypothèse de "dualisation » ou "polarisation » du marché du travail portée par le réseau de recherche international et pluridisciplinaire "Marché du travail et genre » (MAGE) depuis sa création est d'une étonnante actualité. Alors que les entreprises et administrations sont marquées par des réorganisations et restructurations perpétuelles, des réductions d'effectifs et de moyens, qui se sont accélérées depuis la grande récession de 2008, les changements organisationnels, externalisations, acquisitions, fusions, relocalisation ou transferts d'activités, sont rarement examinées à l'aune de leur impact sur les inégalités [Bory et Pochic, 2014]. Les thèmes des discriminations et des restructurations sont rarement étudiés de concert. Or ces décisions ont un impact disproportionné sur les parcours et conditions des femmes, notamment sur le salariat d'exécution. Les employées et les ouvrières, plus souvent membres de minorités ethniques, sont de moins en moins souvent salariées des grands groupes, mais sont reléguées plutôt à leur périphérie, chez les sous-traitants ou dans les filiales, et sur des statuts précaires, avec une faible présence syndicale. Et alors que la fonction publique est un secteur d'emploi très féminisé, surtout à sa base (près de $70 \%$ des catégories $C$ sont féminisées en France), la réduction de l'emploi public et les réformes inspirées par la Nouvelle gestion publique restreignent les opportunités de promotions internes, dégradent les conditions de travail et d'emploi des services, au nom d'une "bonne gestion » en période d'austérité.

Si les femmes dirigeantes, du public comme du privé, blanches et diplômées, sont désormais plus attentives à l'égalité et au sexisme pour elles et leurs consœurs, elles sont souvent moins sensibles aux difficultés de celles qu'elles commandent. Elles peuvent même être des actrices zélées des réorganisations, qui recréent des inégalités en bas de l'échelle, et à la périphérie des organisations. Les restructurations financières peuvent ouvrir des opportunités à celles qui portent la "logique du marché ", sans s'émouvoir du coût social élevé de cette politique de rationalisation des coûts. Nous retrouvons ici l'analyse de Judy Wajcman [Wajcman, 1998] : la performance de genre des femmes cadres supérieures dans les restructurations s'avère en pratique très loin de la rhétorique du "management au féminin » qui serait caractérisée par l'écoute, la proximité, la prévenance et l'attention à autrui. "L'indifférence des privilégiés » [Tronto, 2009] ne s'exerce pas qu'à l'égard de leur personnel de maison, ou des travailleuses des métiers du soin, souvent étrangères ou racisées. On le retrouve, sous une autre forme, au sein de leurs entreprises, notamment à l'égard des femmes nonq cadres, pourtant souvent diplômées, mais n'ayant pas le même 
" potentiel " ou "talent», surtout si elles réalisent leur activité de travail à distance du siège. Comme l'origine populaire, la "féminité respectable" peut devenir une ressource dans le milieu professionnel, à condition d'avoir intégré tous les codes des grands patrons, notamment l'idéologie néolibérale, le carriérisme, le sur-travail et les réseaux d'influence [Naudet, 2014]. Ce faisant, ces femmes participent à l'illusion de la méritocratie et à la défense des privilèges, puisque la féminité ne peut devenir une ressource que pour une petite élite de femmes cooptées.

La féminisation de la gouvernance des grandes organisations n'a donc pas d'effets en cascade sur la promotion des femmes des étages inférieurs. La théorie du "role model » (selon laquelle un des freins aux carrières des femmes serait leur absence de référence inspirante) ne fonctionne que si les personnes peuvent s'identifier à leurs supérieures et font face à la même structure d'opportunités [Kanter, 1977]. Les femmes non-cadres, concentrées dans des services tertiaires ou isolées dans des unités techniques, et qui ne sont associées à aucune formation ou événement organisés sur l'égalité, la parentalité ou la mixité, s'avèrent parfois moins "conscientisées " sur le sujet que leurs cheffes. Surtout si elles ne sont pas syndiquées, elles utilisent rarement ce prisme des rapports de genre ou du sexisme pour comprendre les obstacles professionnels qu'elles rencontrent. Pourtant, elles rencontrent des difficultés de progression en interne, des carrières plates et parfois chaotiques et le " plafond de verre » du passage au statut de cadre est encore plus net pour elles. Le fait que la stratégie financière et les décisions de réorganisation passent désormais souvent par des cheffes femmes, contribue sans doute à brouiller l'appréhension des rapports sociaux de domination. Cela trouble la "classe de sexe ", tout en rendant plus perceptibles les divisions de classe qui s'accentuent au sein des grandes entreprises, et plus largement au sein du salariat.

Si la comparaison avec d'autres pays est essentielle, il me semble que nos cadres d'analyse ne doivent plus être cloisonnés par des frontières nationales pour mieux intégrer les effets de la globalisation économique. En ce domaine, les sociologues du travail et du genre prennent progressivement la mesure du rôle majeur des multinationales dans la division internationale du travail et dans l'externalisation des inégalités "pour le bas » en dehors de leur périmètre juridique, vers leurs sous-traitants et leurs filiales, du Nord vers les pays de l'Est ou les pays du Sud, territoires où le coût salarial est bien plus bas, les contextes légaux sont moins contraignants et les syndicats moins bien implantés. Les rares enquêtes existantes permettent d'éclairer une stratégie que les grandes entreprises conceptualisent rarement sous cette forme, celle des bénéfices économiques de l'inégalité sexuée et de la sous-valorisation du travail féminin [Falquet et al., 2010]. L'idéal serait évidemment de 
pouvoir faire des enquêtes multi-situées au sein des multinationales, pour voir comment au sein d'un même groupe ou le long de la " chaîne du produit ", les questions de l'égalité et des droits des femmes se déclinent en fonction des contextes culturels et légaux. L'enjeu scientifique et politique est de voir comment, et à quelles conditions, des normes d'égalité peuvent circuler (ou non) dans ces espaces transnationaux, et d'identifier les zones prioritaires d'action pour résorber ces inégalités sexuées produites par le marché.

\section{Bibliographie}

ACKER Joan, 2009, "From glass ceiling to inequality regimes ", Sociologie du Travail, $\mathrm{n}^{\circ} 51$, p. $199-217$.

Bender Anne-Françoise, Berrebi-Hoffmann Isabelle et Reigne Philippe, 2015, " Les quotas de femmes dans les conseils d'administration ", Travail, Genre et Sociétés, n², p. 169-173.

BERENI Laure, 2015, La bataille de la parité. Mobilisations pour la féminisation du pouvoir, Paris, Economica.

BERENI Laure, 2009, “'Faire de la diversité une richesse pour l'entreprise'. La transformation d'une contrainte juridique en catégorie managériale ", Raisons Politiques, n³5, p. 87-105.

Berrey Ellen, 2014, "Breaking glass ceiling, ignoring dirty floor: The culture and class bias of diversity management ", American Behavioral Scientist, vol. 58, n² 2, p. 347-370.

Blanchard Soline, Boni-Le Goff Isabel et Rabier Marion, 2013, "Une cause de riches ? L'accès des femmes au pouvoir économique ", Sociétés Contemporaines, $n^{\circ} 89$, p. 101-129.

BonI-LE Goff Isabel, 2010, "Au nom de la diversité : analyse écologique du développement des réseaux de femmes cadres en France ", Sociologies Pratiques, n²1, p. 83-95.

BonI-LE Goff Isabel, 2013, Le sexe de l'expert, Régimes de genre et dynamique des inégalités dans l'espace du conseil en management, Thèse en sociologie, EHESS.

BORY Anne et PochIc Sophie (dir.), 2014, « Dossier : Une crise sans précédent ? Expériences et contestations des restructurations (I et II) ॥, Travail et Emploi, n 137 et 138.

Charpenel Marion, Demilly Hélène et Pochic Sophie, 2017, "Egalité négociée, égalité standardisée ", Travail, Genre et Sociétés, n³7, p. 169-173

CoCKBURN Cynthia, 1991, In the way of women: Men's resistance to sex equality in organizations, Basingstoke, Macmillan.

CONNELL Raewyn, 2005, "Change among the gatekeepers: Men, masculinities and gender equality in the global arena ", Signs, vol. 30, n 3, p. 1801-1825.

DeLPHY Christine, 2001, L'ennemi principal (Tome 2) : penser le genre, Paris, Syllepse.

DJELIC Marie-Laure, 2004, "L'arbre banian de la mondialisation ", Actes de la Recherche en Sciences Sociales, $n^{\circ} 1$, p. 107-113.

EISENSTEIN Hester, 2009, Feminism seduced: How global elites use women's labor and ideas to exploit the world, Boulder, Paradigm Publishers. 
FAlquet Jules, HiRATA Helena et al., 2010, Le sexe de la mondialisation : genre, classe, race et nouvelle division du travail, Paris, Presses de Sciences Po.

FRASER Nancy, 2011, "Féminisme, capitalisme et ruses de l'histoire », Cahiers du genre, n 1 , p. 165-192.

Guillaume Cécile, 2018. Syndiquées. Défendre les intérêts des femmes au travail, Paris, Presses de Sciences Po.

JACQuemart Alban, Le MAnca Fanny et Pochic Sophie, 2016, «Femmes hautes fonctionnaires en France, l'avènement d'une égalité élitiste ? ", Travail, Genre et Sociétés, n 35, p. 27q 45. JACQUOT Sophie, 2014, L'égalité au nom du marché ? Emergence et démantèlement de la politique européenne d'égalité entre les hommes et les femmes, Bruxelles, Peter Lang.

KANTER Moss Rosabeth, 1977, Men and Women in the Corporation, New York, Basic Books.

KANTOLA Johanna et SQUIRES Judith, 2012, "From state feminism to market feminism?", International Political Science Review, vol. 33, $n^{\circ} 4$, p. 382q 400.

KIRTON Gill et Greene Anneq Marie, 2005, The dynamics of managing diversity: A critical approach, Oxford, Elsevier Butterworthq Heinemann.

LAUFER Jacqueline, 2014, L'égalité professionnelle entre les hommes et les femmes, Paris, La Découverte.

LAUFER Jacqueline et SILVERA Rachel, 2017, « Controverse : Des lois à la négociation... Quoi de neuf pour l'égalité ? », Travail, Genre et Sociétés, n³7, p.129q 169.

MARUANI Margaret, 1979, Les syndicats à l'épreuve du féminisme, Paris, Syros q CNRS.

Meynaud Hélèneq Yvonne, Fortino Sabine et Calderon José (dir.), 2009, « Dossier: La mixité au service de la performance économique ", Cahiers du Genre, $\mathrm{n}^{\circ} 47$.

NAUDET Jules, 2012, Entrer dans l'élite. Parcours de réussite en France, aux Étatss Unis et en Inde, Paris, Presses Universitaires de France.

PRÜGL Elisabeth, 2015, "Neoliberalising feminism », New Political Economy, vol. 20, n 4, p. $614 q 631$.

PRÜGL Elisabeth et TRUE Jacqui, 2014, « Equality means business? Governing gender through transnational publicq private partnerships », Review of International Political Economy, vol. 21, nº, p. 1137q 1169.

RABIER Marion, 2013, Entrepreneuses de cause. Contribution à une sociologie des engagements des dirigeantes économiques en France, Thèse de sociologie, EHESS.

ROBERTS Adrienne, 2012, "Financial crisis, financial firms... And financial feminism? The rise of 'transnational business feminism' and the necessity of marxistq feminist IPE ", Socialist Studies/Études socialistes, vol. 8, n², p. 85q 108.

SENAC Réjane, 2015, L'égalité sous conditions. Genre, parité, diversité dans une république néolibérale, Paris, Presses de Sciences Po.

Tronto Joan, 2009, Un monde vulnérable. Pour une politique du Care, Paris, La Découverte.

WAJCMAN Judy, 1998, Managing like a man: Women and men in corporate management, Pennsylvania State University Press. 